\section{Research Square}

Preprints are preliminary reports that have not undergone peer review. They should not be considered conclusive, used to inform clinical practice, or referenced by the media as validated information.

\title{
Influence of COL9A1 and COL19A1 Polymorphisms on Kaschin-Beck Disease Risk
}

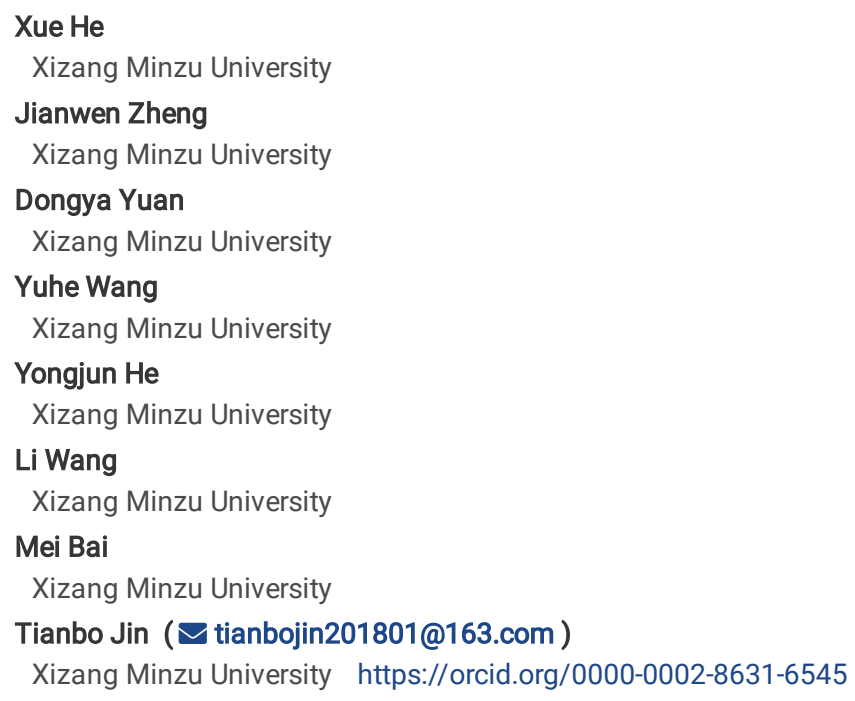




\section{Abstract}

Objective We aimed to determine whether COL9A1 and COL19A1 polymorphisms were associated with Kaschin-Beck disease (KBD) risk.

Methods Five single nucleotide polymorphisms (SNPs) in COL9A1 and COL19A1 were genotyped in 316 KBD patients and 320 healthy controls using the Agena MassARRAY platform. The association between genetic polymorphisms ( COL9A1 : rs3806093, rs603410 and rs621347; COL19A1 : rs9346371 and rs555313) and KBD risk were assessed using logistic regression model by calculating odds ratio (OR) and 95\% confidence interval (CI).

Results After adjustment with age and sex, the frequency distributions of genotypes in rs3806093 and rs9346371 were significantly different between cases and controls. COL9A1 rs3806093 significantly increased $\mathrm{KBD}$ risk in co-dominant $(\mathrm{OR}=14.80,95 \% \mathrm{Cl}=1.42-154.80, \mathrm{p}=0.024)$ and recessive $(\mathrm{OR}=$ $16.39,95 \% \mathrm{Cl}=1.60-168.20, \mathrm{p}=0.019)$ models. Meanwhile, $\mathrm{COL9A} 1 \mathrm{rs} 555313$ was associated with $\mathrm{KBD}$ risk in recessive model $(\mathrm{OR}=3.80,95 \% \mathrm{Cl}=1.01-$ $14.27, \mathrm{p}=0.048$ ). In addition, haplotype analysis revealed two blocks (block 1: rs3806093, rs603410 and rs621347; block 2: rs9346371 and rs555313).

Conclusion COL9A1 and COL19A1 polymorphisms were associated with KBD risk in the Chinese Han population, suggesting roles of COL9A1 and COL19A1 in the development of KBD.

\section{Introduction}

Kaschin-Beck disease (KBD) is a chronic osteochondropathy, characterized by cartilage degeneration, chondrocyte necrosis and apoptosis(1, 2). KBD mainly distributed from southeastern Siberia to China. There are approximately 690,000 KBD patients and more than 10 million people are likely to suffer from KBD in China(3). The etiology of KBD remains unclear, studies suggest that KBD is a complex disease made by interactions between environmental factors and genetic factors(4). More than $40 \%$ of KBD risk could attribute to genetic components(3). Certain susceptibility genes may have effects on KBD risk.

Collagens are the most abundant proteins, the collagen family comprises 28 members $(I-X X V I I I)(5,6)$. One of the three alpha chains of type IX collagen are coded by COL9A1 gene, which is essential for the functional longevity of joint cartilages and connected with osteochondropathy(7, 8). Studies in knockout mice have shown that lack of type IX collagen is associated with early onset osteoarthritis. Mutations in COL9A1 are associated with osteoarthritis, lumbar disc disease, and multiple epiphyseal dysplasia(9-11). Specially, it is reported that COL9A1 polymorphism (rs6910140) was significantly associated with KBD risk in a northwest Chinese Han population(12). COL19A1encodes the alpha chain of type XIX collagen, a member of the fibril-associated collagens with interrupted helices (FACIT) collagen family. COL19A1 was localized to 6q12-q14, the same region of COL9A1 gene(13). Type XIX collagen was involved in the initial stages of skeletal muscle cell differentiation(14). Studies reported that Type XIX collagen may contribute to brain disorders(15), but no data on the relationship between COL19A1 polymorphisms and KBD risk.

In this study, we aimed to investigate whether polymorphisms of COL9A1 and COL19A1 affect the risk of KBD. We conducted a case-control study and focused on five polymorphisms (rs3806093, rs603410 and rs621347 of COL9A1; rs934631 and rs555313 of COL 19A1) to assess the associations between genetic polymorphisms and KBD risk.

\section{Methods}

Study subjects

A total of 636 Chinese Han individuals (316 KBD patients, 320 healthy controls) were recruited from Affiliated Hospital of Xizang Minzu University, Xianyang, China. According to the national diagnostic criteria of China (WS/T 207-2010), all KBD patients was diagnosed by two KBD experts. The exclusion criteria included people who had clinical symptoms or radiographic changes of other osteochondropathy. The healthy controls were randomly collected from disease-free individuals who had health examination in the Affiliated Hospital of Xizang Minzu University. This study was in the Declaration of Helsinki and approved by the Ethics Committee of Affiliated Hospital of Xizang Minzu University, and we obtained written informed consents from all study subjects before study.

Genotyping

Based on the data of the Han Chinese population in Beijing (CHB) from the 1000 Genomes project and previous studies(4), we selected three SNPs (rs3806093, rs603410 and rs621347) of COL9A1 gene and two SNPs (rs9346371 and rs555313) of COL 19A1, with minor allele frequency (MAF) greater than $5 \%$. Genomic DNA was extract from whole blood using blood DNA kit (GoldMag Co. Ltd., Xi囚an, China) and was measured by Nanodrop 2000 (Thermo Scientific, Waltham, Massachusetts, USA).We genotyped five SNPs of COL9A1 and COL 19A1 using Agena MassARRAY platform (Agena, San Diego, CA, USA). The Agena MassARRAY Assay Design 3.0 Software (San Diego, CA USA) was used to design the primers for each SNP (Table 1). Additionally, we managed and analyzed data by the Agena Typer 4.0 Software (San Diego, CA, USA)(16).

\section{Statistical analysis}

Statistical analysis was conducted with SPSS 21.0 (IBM®, Armonk, New York, USA)(17). $P<0.05$ was considered statistically significant. The HardyWeinberg equilibrium (HWE) of each SNP was assessed by Fisher's exact test in the control group. Logistic regression analysis adjusted by age and sex was used to evaluate the association of $C O L 9 A 1$ and $C O L 9 A 1$ polymorphisms with KBD risk through calculating odds ratio (OR) and $95 \%$ confidence 
interval ( $\mathrm{Cl}$ ). Allele model and genetic models (co-dominant, dominant, recessive and additive) were assessed by the chi square test and PLINK software. Then, haplotype analysis was performed by Haploview software (version 4.2) and PLINK software.

\section{Results}

Study subjects

A total of 316 cases (183 men and 133 women) and 320 controls ( 239 men and 81 women) were included in this study. The characteristics of study subjects are presented in Table 2. The mean ages of cases and controls were $54.70 \pm 17.14$ and $19.00 \pm 1.60$ years old, individually. There are significant differences in the distribution of age and sex between two groups.

Association of $C O L 9 A 1$ and $C O L 9 A 1$ polymorphisms with KBD risk

The loci information of five SNPs in COL9A1 and COL19A1 are shown in Table3. All SNPs are in HWE and the MAFs in two groups are listed in this table. The association of COL9A1 and COL 19A1 polymorphisms with KBD risk are presented in Table 4 . The distribution frequencies of genotypes in COL9A1 rs3806093 and COL19A1 rs9346371 are significantly different between the two groups. Rs3806093 is significantly associated with higher risk of KBD in codominant $(\mathrm{OR}=14.80,95 \% \mathrm{Cl}=1.42-154.80, p=0.024)$ and recessive $(\mathrm{OR}=16.39,95 \% \mathrm{Cl}=1.60-168.20, p=0.019)$ models. Individuals with $\mathrm{TT}$ genotype of rs9346371 are associated with increased KBD risk compared with TC-CC genotype $(\mathrm{OR}=3.80,95 \% \mathrm{Cl}=1.01-14.27, p=0.048)$. There are no significant associations between other SNPs and KBD risk $(p>0.05)$.

Haplotype analysis

We performed the haplotype analysis of $C O L 9 A 1$ and $C O L 19 A 1$ polymorphisms with KBD risk (Table 5). There are no significant relationship between COL9A1 and COL 19A1 polymorphisms and KBD risk $(p>0.05)$. As shown in Figure 1 and Figure 2, we observed two blocks (block 1: rs3806093, rs603410 and rs621347; block 2: rs9346371 and rs555313).

\section{Discussion}

In this study, three SNPs in COL9A1 (rs3806093, rs603410 and rs621347) and two SNPs in COL $19 A 1$ (rs9346371 and rs555313) were included to explore the association of genetic polymorphisms and risk of KBD. The results showed that rs3806093 of COL9A1 and rs9346371 of COL 19A1 were significantly associated with increased KBD risk.

KBD is an endemic multiple and deformed osteoarthropathy(18). The electron microscopic analysis have showed that a reduction in the collagen fibril diameter and a loss of the fibril banding patterns in the cartilage matrix among the KBD patients(19). Type IX collagen plays a vital role in the degradation of cartilage and bone(12). Several studies have revealed that genes polymorphisms encoding type IX collagen had relationship with osteoarthropathy risk $(8,20)$. Previous study genotyped fifteen SNPs in COL9A1 and found rs6910140 of COL9A1 plays an important role in the risk and severity of KBD in the Chinese Han population(12). Our data confirmed the association between COL9A1 gene and KBD risk. In co-dominant and recessive models, rs3806093 of COL9A1 had a strong association with KBD susceptibility $(p<0.05)$. COL 19A1 encodes type XIX collagen, which mainly expressed in central neurons and is necessary for hippocampal synapses formation(21). In this study, we assessed the association between COL $19 A 1$ polymorphisms and KBD risk. We observed that rs 9346371 of $C O L 19 A 1$ was associated with the risk of KBD in recessive model. Our results suggest that polymorphisms of $C O L 9 A 1$ and COL19A1may be involved in the development of KBD. However, further studies are needed to explore the exact mechanism of genetic polymorphisms in KBD.

Some limitations should also be considered. First, the relatively small sample size in this study. Second, we cannot do stratification analysis due to the lack of information on subjects. Third, many environmental factors and lifestyle could affect the susceptibility of KBD, we could not eliminate all factors in this study. Hence, larger sample size and well designed studies are required to validate the association of COL9A1 and COL 19A1 polymorphisms with KBD risk.

\section{Conclusion}

In conclusion, we found polymorphisms of COL9A1 and COL 19A1 were associated with the risk of KBD, suggesting the role of COL9A1 and COL $19 A 1$ polymorphisms in the development of KBD. Further studies are required to validate the influence of COL9A1 and COL 19A1 polymorphisms on KBD risk.

\section{Declarations}

\section{Ethics approval and consent to participate}

This study was in the Declaration of Helsinki and approved by the Ethics Committee of Affiliated Hospital of Xizang Minzu University, and we obtained written informed consents from all study subjects before study.

\section{Competing interests}

The authors declare that they have no competing interests

\section{Consent for publication}


Not applicable.

\section{Funding}

This research received no specific grant from any funding agency in the public, commercial, or not-for-profit sectors.

\section{Authors contribution}

$\mathrm{XH}$ drafted the manuscript. JWZ and YHW performed the DNA extraction and genotyping; LW and MB performed the data analysis; DYY and MB performed the sample collection and information recording; $\mathrm{XH}$ and TBJ conceived and supervised the study.

\section{Acknowledgement}

We thank all the people involved in our study.

\section{Reference}

1.Shi Y, Lu F, Liu X, Wang Y, Huang L, Liu X, et al. Genetic variants in the HLA-DRB1 gene are associated with Kashin-Beck disease in the Tibetan population. Arthritis \& Rheumatism. 2011;63(11):3408-16.

2.Wang SJ, Guo X, Zuo H, Zhang YG, Xu P, Ping ZG, et al. Chondrocyte apoptosis and expression of Bcl-2, Bax, Fas, and iNOS in articular cartilage in patients with Kashin-Beck disease. The Journal of rheumatology. 2006;33(3):615-9.

3.Lü A, Guo X, Aisha M, Shi X, Zhang YZ, Zhang Y. Kashin-Beck disease and Sayiwak disease in China: Prevalence and a comparison of the clinical manifestations, familial aggregation, and heritability. Bone. 2011;48(2):347-53.

4.Zhang F, Guo X, Wang W, Yan H, Li C. Genome-wide gene expression analysis suggests an important role of hypoxia in the pathogenesis of endemic osteochondropathy Kashin-Beck disease. PloS one. 2011;6(7):e22983.

5.Ricard-Blum S. The collagen family. Cold Spring Harbor perspectives in biology. 2011;3(1):a004978.

6.Jiang X, Wang Y, Fan D, Zhu C, Liu L, Duan Z. A novel human-like collagen hemostatic sponge with uniform morphology, good biodegradability and biocompatibility. Journal of biomaterials applications. 2017;31(8):1099-107.

7.Bönnemann CG, Cox GF, Shapiro F, Wu J-J, Feener CA, Thompson TG, et al. A mutation in the alpha 3 chain of type IX collagen causes autosomal dominant multiple epiphyseal dysplasia with mild myopathy. Proceedings of the National Academy of Sciences. 2000;97(3):1212-7.

8.Czarny-Ratajczak M, Lohiniva J, Rogala P, Kozlowski K, Perälä M, Carter L, et al. A mutation in COL9A1 causes multiple epiphyseal dysplasia: further evidence for locus heterogeneity. The American Journal of Human Genetics. 2001;69(5):969-80.

9.Lohiniva J, Paassilta P, Seppänen U, Vierimaa O, Kivirikko S, Ala-Kokko L. Splicing mutations in the COL3 domain of collagen IX cause multiple epiphyseal dysplasia. American journal of medical genetics. 2000;90(3):216-22.

10.Mustafa Z, Chapman K, Irven C, Carr A, Clipsham K, Chitnavis J, et al. Linkage analysis of candidate genes as susceptibility loci for osteoarthritissuggestive linkage of COL9A1 to female hip osteoarthritis. Rheumatology. 2000;39(3):299-306.

11.Jakkula E, Melkoniemi M, Kiviranta I, Lohiniva J, Räinä S, Perälä M, et al. The role of sequence variations within the genes encoding collagen II, IX and XI in non-syndromic, early-onset osteoarthritis. Osteoarthritis and cartilage. 2005;13(6):497-507.

12.Shi X, Zhang F, Lv A, Wen Y, Guo X. COL9A1 gene polymorphism is associated with Kashin-Beck disease in a northwest Chinese Han population. PloS one. 2015;10(3):e0120365.

13.Khaleduzzaman M, Sumiyoshi H, Ueki Y, Inoguchi K, Ninomiya Y, Yoshioka H. Structure of the human type XIX collagen (COL19A1) gene, which suggests it has arisen from an ancestor gene of the FACIT family. Genomics. 1997;45(2):304-12.

14.Sumiyoshi H, Laub F, Yoshioka H, Ramirez F. Embryonic expression of type XIX collagen is transient and confined to muscle cells. Developmental dynamics: an official publication of the American Association of Anatomists. 2001;220(2):155-62.

15.Su J, Cole J, Fox MA. Loss of interneuron-derived collagen XIX leads to a reduction in Perineuronal nets in the mammalian telencephalon. ASN neuro. 2017;9(1):1759091416689020.

16.Zhang F, Guo X, Duan C, Wu S, Yu H, Lammi M. Identification of differentially expressed genes and pathways between primary osteoarthritis and endemic osteoarthritis (Kashin-Beck disease). Scandinavian journal of rheumatology. 2013;42(1):71-9.

17.Wang X, Ning Y, Tan W, Yu H, Li Z, Guo X. Population-based comparative analysis of differentially expressed genes between Kashin-Beck disease grades I and II. Scandinavian journal of rheumatology. 2016;45(3):230-5. 
18.Wang W-Z, Guo X, Duan C, Ma WJ, Zhang Y, Xu P, et al. Comparative analysis of gene expression profiles between the normal human cartilage and the one with endemic osteoarthritis. Osteoarthritis and Cartilage. 2009;17(1):83-90.

19.Zhang G, Liu J, Yu J, Shi Y, Zhang S. Ultrastructure of chondrocytes in articular cartilage of Kashin-Beck disease. Chin J Endemiol. 1989;8:27-30.

20.Jackson GC, Marcus-Soekarman D, Stolte-Dijkstra I, Verrips A, Taylor JA, Briggs MD. Type IX collagen gene mutations can result in multiple epiphyseal dysplasia that is associated with osteochondritis dissecans and a mild myopathy. American Journal of Medical Genetics Part A. 2010;152(4):863-9.

21.Su J, Gorse K, Ramirez F, Fox MA. Collagen XIX is expressed by interneurons and contributes to the formation of hippocampal synapses. Journal of Comparative Neurology. 2010;518(2):229-53.

\section{Tables}

Table 1 Primers used in the study

\begin{tabular}{|c|c|c|c|c|c|}
\hline Gene & SNP & $1^{\text {st_-PCRP }}$ & $2^{\text {nd-PCRP }}$ & UEP_DIR & UEP_SEQ \\
\hline \multirow[t]{3}{*}{ COL9A1 } & 3806093 & ACGTTGGATGAAGGACTCCAGTTTGAACAC & ACGTTGGATGTGAACATCTAGGTTTCTGAC & $\mathrm{R}$ & TTCAAACACAACAC \\
\hline & 10 & ACGT' & CAGT & $\mathrm{R}$ & $\mathrm{CTT}$ \\
\hline & rs621347 & ACGTTGGATGCCAGGACAACATGTTAGGAC & ACGTTGGATGGTCAGATGTTACAGTAACAC & $\mathrm{F}$ & agTATGAAATAACTATGCAGAAAACC \\
\hline \multirow[t]{2}{*}{ COL19A1 } & rs9346371 & ACGTTGGATGCTTGTATATGGAAATCACAG & ACGTTGGATGAACTCTTGTTTGCACTCCAG & $\mathrm{F}$ & gACAAAATAATTATGGGCCATGA \\
\hline & rs555313 & ACGTTGGATGTGGGTAATTGGCTTCTGCAC & ACGTTGGATGGGTTCAGTCAGCTTGAAATG & $\mathrm{F}$ & TCCAAATAATCAAATCGATCA \\
\hline
\end{tabular}

SNP, single nucleotide polymorphism; PCRP, polymerase chain reaction primer; UEP, unextended mini sequencing primer; DIR, direction; SEQ, sequence

Table 2 Characteristics of study subjects

\begin{tabular}{|c|c|c|c|}
\hline Characteristics & Cases $(\mathrm{N}=316)$ & Controls $(\mathrm{N}=320)$ & $P$ value \\
\hline Age & $54.70 \pm 17.14$ & $19.00 \pm 1.60$ & $<0.001$ \\
\hline Sex & & & $<0.001$ \\
\hline Man & $183(57.91 \%)$ & 239 (74.69\%) & \\
\hline Woman & 133 (42.09\%) & 81 (25.31\%) & \\
\hline
\end{tabular}

Table 3 The loci information of five SNPs in COL9A1 and COL19A1

\begin{tabular}{lccccccc}
\hline Gene & SNP & Chromosome position & Alleles & SNP location & MAF (cases) & MAF (controls) & HWE test ( $P$ ) \\
\hline COL9A1 & rs3806093 & 6: 70273226 & A/G & intron & 0.163 & 0.166 & 0.550 \\
\hline & rs603410 & 6: 70274945 & T/G & intron & 0.214 & 0.214 & 1.000 \\
\hline & rs621347 & $6: 70276646$ & A/G & intron & 0.375 & 0.381 & 1.000 \\
\hline COL19A1 & rs9346371 & 6: 70210157 & T/C & 3'UTR & 0.349 & 0.361 & 0.278 \\
& rs555313 & 6: 70214317 & T/C & 3'UTR & 0.465 & 0.441 & 1.000 \\
\hline
\end{tabular}

SNP, single nucleotide polymorphism; MAF, minor allele frequency; HWE, Hardy-Weinberg equilibrium

Table 4 Association of COL9A1 and COL19A1 polymorphisms with Kaschin-Beck disease risk 


\begin{tabular}{|c|c|c|c|c|c|c|}
\hline SNP & Genotype & Count (case) & Count (control) & Model & OR (95\%CI) & $P$ \\
\hline \multirow[t]{9}{*}{ rs3806093 } & $\mathrm{A} / \mathrm{G}$ & $103 / 529$ & $106 / 534$ & Allele & $0.98(0.73-1.32)$ & 0.899 \\
\hline & $\mathrm{AA}$ & 8 & 7 & Co-dominant & $14.80(1.42-154.80)$ & 0.024 \\
\hline & AG & 87 & 92 & & $0.68(0.19-2.38)$ & 0.542 \\
\hline & GG & 221 & 221 & & 1 & \\
\hline & AA-AG & 95 & 99 & Dominant & $1.04(0.34-3.15)$ & 0.944 \\
\hline & GG & 221 & 221 & & 1 & \\
\hline & $\mathrm{AA}$ & 8 & 7 & Recessive & $16.39(1.60-168.20)$ & 0.019 \\
\hline & AG-GG & 308 & 313 & & 1 & \\
\hline & & & & Additive & $1.51(0.59-3.87)$ & 0.386 \\
\hline \multirow[t]{9}{*}{ rs603410 } & $\mathrm{T} / \mathrm{G}$ & $135 / 497$ & $135 / 497$ & Allele & $1.00(0.76-1.31)$ & 1.000 \\
\hline & TT & 15 & 14 & Co-dominant & $0.41(0.01-11.54)$ & 0.599 \\
\hline & TG & 105 & 107 & & $0.70(0.24-2.05)$ & 0.517 \\
\hline & GG & 196 & 195 & & 1 & \\
\hline & TT-TG & 120 & 121 & Dominant & $0.68(0.24-1.94)$ & 0.469 \\
\hline & GG & 196 & 195 & & 1 & \\
\hline & TT & 15 & 14 & Recessive & $0.47(0.02-12.43)$ & 0.653 \\
\hline & TG-GG & 301 & 302 & & 1 & \\
\hline & & & & Additive & $0.68(0.26-1.77)$ & 0.434 \\
\hline \multirow[t]{9}{*}{ rs 621747} & $\mathrm{~A} / \mathrm{G}$ & $237 / 395$ & $244 / 396$ & Allele & $0.97(0.78-1.22)$ & 0.818 \\
\hline & $\mathrm{AA}$ & 47 & 46 & Co-dominant & $1.37(0.35-5.35)$ & 0.649 \\
\hline & $\mathrm{AG}$ & 143 & 152 & & $0.49(0.16-1.49)$ & 0.206 \\
\hline & GG & 126 & 122 & & 1 & \\
\hline & AA-AG & 190 & 198 & Dominant & $0.66(0.25-1.78)$ & 0.411 \\
\hline & GG & 126 & 122 & & 1 & \\
\hline & $\mathrm{AA}$ & 47 & 46 & Recessive & $1.93(0.54-6.88)$ & 0.313 \\
\hline & AG-GG & 269 & 274 & & 1 & \\
\hline & & & & Additive & $0.97(0.48-1.99)$ & 0.941 \\
\hline \multirow[t]{3}{*}{ rs9346371 } & $\mathrm{T} / \mathrm{C}$ & $219 / 409$ & $231 / 409$ & Allele & $0.95(0.75-1.19)$ & 0.650 \\
\hline & $\mathrm{TT}$ & 33 & 37 & Co-dominant & $3.69(0.85-16.00)$ & 0.081 \\
\hline & TC & 153 & 157 & & $0.95(0.31-2.93)$ & 0.929 \\
\hline
\end{tabular}




\begin{tabular}{|c|c|c|c|c|c|c|}
\hline & $\mathrm{CC}$ & 128 & 126 & & 1 & \\
\hline & TT-TC & 186 & 194 & Dominant & $1.27(0.45-3.58)$ & 0.649 \\
\hline & $\mathrm{CC}$ & 128 & 126 & & 1 & \\
\hline & $\mathrm{TT}$ & 33 & 37 & Recessive & $3.80(1.01-14.27)$ & 0.048 \\
\hline & TC-CC & 281 & 283 & & 1 & \\
\hline & & & & Additive & $1.68(0.77-3.66)$ & 0.192 \\
\hline \multirow[t]{9}{*}{ rs555313 } & $\mathrm{T} / \mathrm{C}$ & $294 / 338$ & $282 / 358$ & Allele & $1.10(0.89-1.38)$ & 0.379 \\
\hline & $\mathrm{TT}$ & 74 & 62 & Co-dominant & $0.30(0.06-1.56)$ & 0.152 \\
\hline & $\mathrm{TC}$ & 146 & 158 & & $0.48(0.15-1.50)$ & 0.205 \\
\hline & $\mathrm{CC}$ & 96 & 100 & & 1 & \\
\hline & TT-TC & 210 & 220 & Dominant & $0.44(0.15-1.31)$ & 0.139 \\
\hline & $\mathrm{CC}$ & 96 & 100 & & 1 & \\
\hline & $\mathrm{TT}$ & 74 & 62 & Recessive & $0.50(0.12-2.09)$ & 0.345 \\
\hline & TC-CC & 242 & 258 & & 1 & \\
\hline & & & & Additive & $0.53(0.24-1.19)$ & 0.124 \\
\hline
\end{tabular}

SNP, single nucleotide polymorphism; OR, odds ratio; CI, confidence interval

Bold data means significant difference $(p<0.05)$

Table 5 Haplotype analysis of COL9A1 and COL19A1 polymorphisms with Kaschin-Beck disease risk

\begin{tabular}{|c|c|c|c|c|c|c|c|c|}
\hline \multirow[t]{2}{*}{ Gene } & \multirow[t]{2}{*}{ SNPs } & \multirow[t]{2}{*}{ Haplotype } & \multirow[t]{2}{*}{ Frequency in cases } & \multirow[t]{2}{*}{ Frequency in controls } & \multicolumn{2}{|c|}{ Without adjustment } & \multicolumn{2}{|c|}{ With adjustment } \\
\hline & & & & & OR $(95 \% \mathrm{CI})$ & $P$ & OR (95\%CI) & $P$ \\
\hline \multirow[t]{3}{*}{ COL9A1 } & rs3806093|rs603410|rs621347 & GTA & 0.212 & 0.216 & $0.98(0.75-1.28)$ & 0.876 & $0.66(0.26-1.69)$ & 0.392 \\
\hline & & AGA & 0.163 & 0.166 & $0.98(0.73-1.32)$ & 0.897 & $1.51(0.59-3.87)$ & 0.386 \\
\hline & & GGG & 0.377 & 0.381 & $0.98(0.78-1.23)$ & 0.865 & $0.97(0.48-1.99)$ & 0.941 \\
\hline \multirow[t]{3}{*}{ COL19A1 } & rs9346371|rs555313 & $\mathrm{CT}$ & 0.463 & 0.441 & $1.09(0.88-1.36)$ & 0.424 & $0.53(0.24-1.19)$ & 0.124 \\
\hline & & TC & 0.347 & 0.361 & $0.94(0.74-1.19)$ & 0.595 & $1.68(0.77-3.66)$ & 0.192 \\
\hline & & $\mathrm{CC}$ & 0.188 & 0.198 & $0.94(0.71-1.23)$ & 0.640 & $1.15(0.48-2.77)$ & 0.750 \\
\hline
\end{tabular}

SNP, single nucleotide polymorphism; OR, odds ratio; CI, confidence interval

\section{Figures}




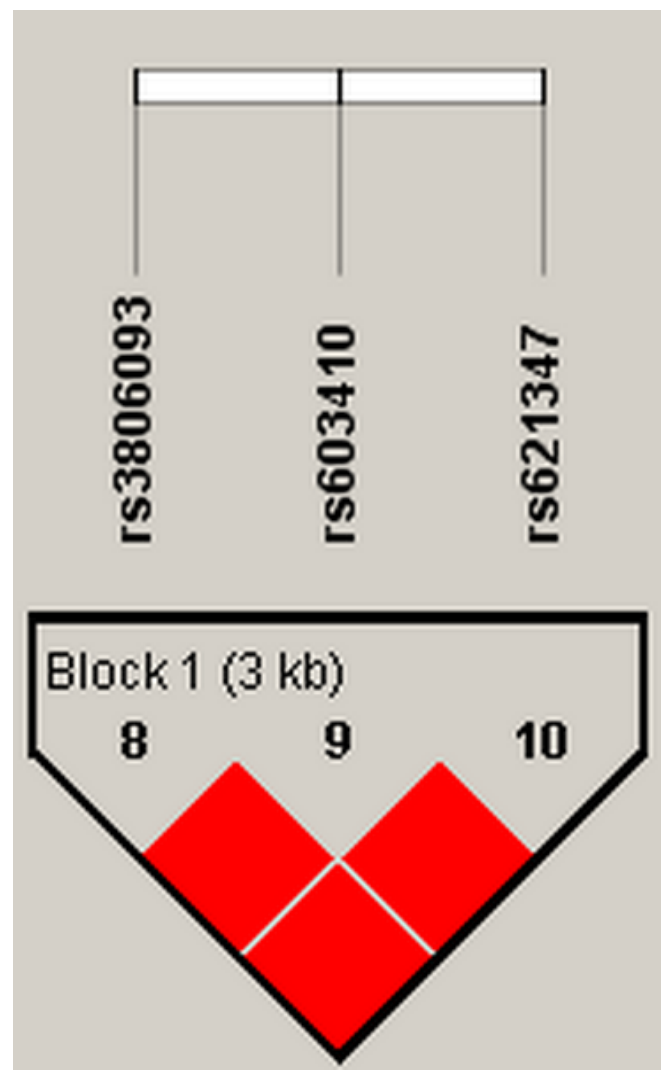

Figure 1

Haplotype block map for the SNPs of COL9A1 Block includes rs3806093, rs603410 and rs621347. The LD between two SNPs is standardized by DQ.

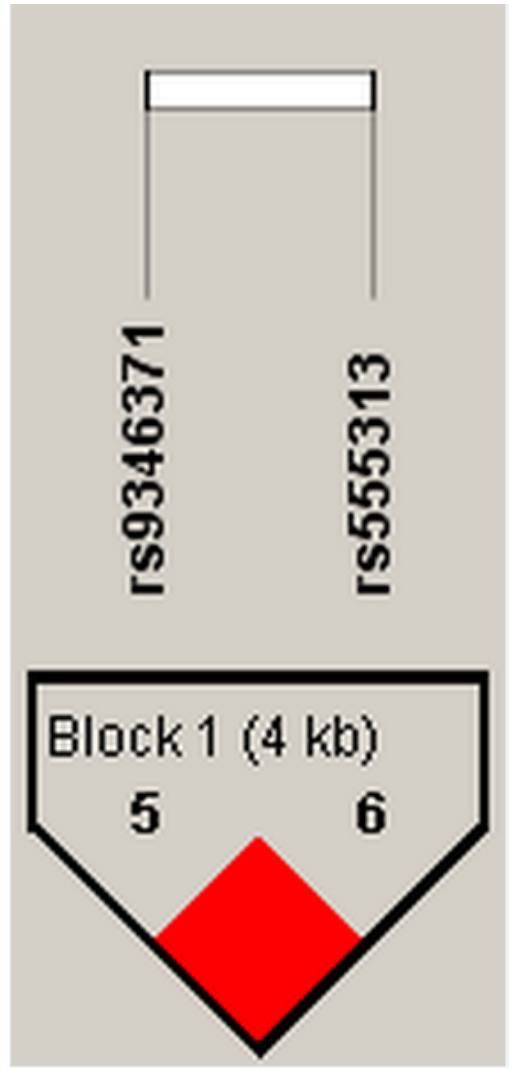

Figure 2

Haplotype block map for the SNPs of COL19A1 Block includes rs9346371 and rs555313. The LD between two SNPs is standardized by DV. 